\title{
Patient Delay and Associated Factors Among Pulmonary Tuberculosis Patients at Hadiya Zone Public Health Facilities in South Ethiopia, 2017
}

\author{
Gedeyon Getahun $^{1}$, Belay Erchafo ${ }^{2,}$, , Lakew Abebe Gebretsadik ${ }^{3}$, Mulugeta Chaka ${ }^{3}$ \\ ${ }^{1}$ Hadiya Zone Health Department, Hossana, Ethiopia \\ ${ }^{2}$ Department of Public Health, College of Medicine and Health Sciences, Wachemo University, Hossana, Ethiopia \\ ${ }^{3}$ Department of Health Education and Behavioral Science, Institute of Health, Faculity of Public Health, Jimma University, Jimma, Ethiopia
}

Email address:

erchafobelay@gmail.com (B. Erchafo)

${ }^{*}$ Corresponding author

\section{To cite this article:}

Gedeyon Getahun, Belay Erchafo, Lakew Abebe Gebretsadik, Mulugeta Chaka. Patient Delay and Associated Factors Among Pulmonary Tuberculosis Patients at Hadiya Zone Public Health Facilities in South Ethiopia, 2017. European Journal of Clinical and Biomedical Sciences. Vol. 4, No. 4, 2018, pp. 55-62. doi: 10.11648/j.ejcbs.20180404.11

Received: September 6, 2018; Accepted: October 4, 2018; Published: November 5, 2018

\begin{abstract}
Early diagnosis and immediate initiation of treatment are essential for an effective TB control program. Delay in treatment is significant to both disease prognoses at the individual level and within the community. Patient delay and associated factors were assessed among pulmonary tuberculosis patients who are on treatment for the first two months at Hadiya zone public health facilities, south Ethiopia, 2017. Facility based cross sectional study triangulated by Qualitative study was employed on 340 Pulmonary Tuberculosis patients from March 10-April 20, 2017. Simple random sampling technique was used to select study health facility. Directly Observed Treatment Short-course User at the beginning of data collection was consecutively recruited in to the study until the intended 340 sample sizes were fulfilled. Data was collected from the participants using a pretested structured interviewer administered questionnaire. Multivariable binary Logistic regressions were used to identify independent predictors of Tuberculosis treatment delay for those variables which are candidate in bivariate analysis. A P-value $<0.05$ at $95 \%$ confidence intervals was considered statistical significance between dependent and predictors variables. Three hundred and Forty PTB patients with a response rate of $97.7 \%$ were enrolled from seven diagnostics and treatment centers. Among 340 Pulmonary Tuberculosis patients enrolled in the study, of which 49.1\% experienced patient delay. The median patient delay was 31. Unable to read and write (AOR 6, 95\% CI (3.11 21.36), Poor knowledge of Tuberculosis (AOR 3.96, 95\% CI (2.286.86), self-treatment (AOR: 2, 95\% CI (1.143.93), and financial constraint (AOR: 2.092, 95\% CI (1.113.945) were the independent predictors of patient delay. Nearly half of the patients seek their first consultation after thirty days cut-off point. Unable to read and write, Poor knowledge of Tuberculosis, self-treatment and financial constraints were found to have association with patient delay. This may lead to continues existence of Tuberculosis cases which probably leads to the emergence of multiple drugs resistant. Implementation of well-designed information education, communication/behavioral change communication strategy for Tuberculosis control program to overcome high prevalence of patient delay.
\end{abstract}

Keywords: Tuberculosis Treatment Delay, Pulmonary Tuberculosis, Patient Delay, Hadiya Zone, Ethiopia

\section{Introduction}

Tuberculosis (TB) is an infectious bacterial disease caused by Mycobacterium TB which commonly affects the lungs but can affect other parts of the body. Though it affects peoples of all ages and sexes, poverty, malnutrition, overcrowding and more recently human immunodeficiency virus/acquired immunodeficiency syndrome (HIV/AIDS) have been known for decades to make some groups more vulnerable to develop the disease $[1,2]$.

Patient delay is the time interval from the onset of 
symptoms of TB until the first visit to any formal health care facility within 30 days as cut off points. World Health Organization classifies person who presents with symptoms or signs suggestive of TB most common a productive cough or unexplained cough with or without other respiratory or constitutional symptoms lasting 2-3 weeks as TB suspect and the patient will seek treatments within the given period, patients presented to modern health facility more than 2-3 weeks considered as patient delay as WHO recommendation. However, studies added extra margin of 7 days to take 30 days as cut-off to assess magnitude of patient delay: a delay $>30$ day $[3,4]$.

With over 2 million of the total global TB cases and between 250 to 400 thousand TB deaths, Africa is burdened with about $25 \%$ of total Global TB cases. The region is home to nine of the 22 high TB burdened countries. The incidence of TB in Africa remain the highest worldwide, the 2012 rate of new infections TB/HIV burden, a major risk factor the progression of the disease, thus a huge challenge to fighting the disease Was 275 per 100,000 populations with total prevalence at 373 per 100,000 populations. These figures far outnumber the global figures that indicate total incidence and prevalence at 127 and 190 per 100,000 populations $[1,5]$.

In 2015, there were an estimated 10.4 million new (incident) TB cases worldwide, of which 5.9 million (56\%) were among men, 3.5 million (34\%) among women and 1.0 million (10\%) among children. People living with HIV accounted for 1.2 million (11\%) of all new TB cases. Six countries accounted for $60 \%$ of the new cases: India, Indonesia, China, Nigeria, Pakistan and South Africa. Global progress depends on major advances in TB prevention and care in these countries [6].

Tuberculosis is a global health concern; nearly one third of the global population is infected with Mycobacterium TB and at risk of developing the disease. More than $90 \%$ of global TB cases and deaths occur in the developing world, where $75 \%$ of cases are in the most economically productive age group. Over $50 \%$ of TB cases occur among people between 15 and 49 years who mostly live in poor and crowded homes. Timely case detection and prompt treatment initiation are best options in TB prevention and control program because $\mathrm{PTB}+$ cases remain the main source of active infection in our communities. TB is a major public health problem in the Horn of Africa with Ethiopia being the most affected where TB cases increase at the rate of $2.6 \%$ each year $[7,8]$.

Ethiopia adopted the internationally recommended TB control strategy, the Directly Observed Treatment Short (DOTS) course, by early 1900s. The major components of this strategy included case detection by sputum smear microscopy and standardized treatment based on TB patient treatment category with active health workers supervision [8]. Despite services and medicines being given free of charge to TB patients and control strategies being in place for over two decades in Ethiopia, the estimated case detection rate of TB remained relatively low, likely indicating under-diagnosis as well as delays in the diagnosis and treatment of TB. That is the patients fail or delayed to seek health care. These may result in increased costs and disease severity in the individuals, as well as increased morbidity and mortality of TB in the community from increased risk of transmission of TB $[4,9,10]$

Delay in diagnosis and treatment of TB patients could be due to patients' delay in seeking health care. There is growing evidence that access to treatment remains difficult for a high number of TB cases. It also leads to an increased period of infectivity in the community. Delay in seeking appropriate TB treatment is a challenge in TB control. Despite of deferent TB control program, still there is low case detection rate which leads to delayed presentation to diagnosis and initiation of treatment in the study area. Little work has been carried out to try to solve the stated problem, or that some work has been done but gaps still exit; hence the need to conduct the study $[11,12]$.

Early detection and timely initiation of anti TB drugs are pre-requisites for a successful TB control program. In this regard, early health seeking action from patients' side very crucial step. Delayed diagnosis of TB results in MDR TB, high infection and a higher mortality. It also leads to an increased period in infectivity in the community. Hence this study was conducted to assess patient delay in TB treatment for effective implementation of the measures in study areas.

\section{Methodology}

\subsection{Study Area and Period}

The study was conducted in Hadiya zone, which is divided in to 10 rural Woreda and two administrative towns with total of 329 kebeles which are 303 of them rural and 26 of them are urban. Hadiya zone hosts a total of $1,573,841$ populations with a total area of $3542.66 \mathrm{Sq}$. Km. in the Zone, TB prevalence rate is $58 \%$. Currently, health care provision within the zone is carried out through 61 health centers, 309 health posts, and 3 public hospitals. There were 131 different privately owned clinics that also rendered health services to the community from March 1, 2017 to April 1, 2017.

\subsection{Study Design and Population}

A Facility based cross-sectional study to investigate factors influencing tuberculosis treatment delays in seeking tuberculosis treatment was employed. All sampled PTB patients registered in NTLP in selected health facilities that were on DOTS, during their intensive phase of treatment was considered as the study population.

\subsection{Sample Size Determination}

The sample size for this study was determined using single population proportion formula by taking $\mathrm{p}$, proportion of patient delay for more than 30 days in Amhara region $(\mathrm{P}=$ $48 \%$ ). Other assumptions made during the sample size calculation, was $5 \%$ margin of error (d) and $95 \%$ confidence interval then the required sample size becomes 383 . Since the expected total number of all form TB cases in the study area is less than 10,000 or $\mathrm{n} / \mathrm{N}$ is $>0.05$, we use correction 
formula. Then the final sample size was 347 .

\subsection{Sampling Technique and Procedures}

Three woreda were selected by simple random sampling from the ten districts whereas, one town administration was included randomly from urban. Simple random sampling technique was used to identify study health facilities to be enrolled for the study. All study facilities were selected proportionally based on the size of the tuberculosis cases per each facility. The sample size was proportionally allocated to each of study health facilities (Hosanna $\mathrm{HC}=40$, Bobicho $\mathrm{HC}=30$, General hospital $=80$, Homacho district hospital $=60$, omocora $\mathrm{HC}=40$, Shone primary hospital $=55$, Korega $\mathrm{HC}=35$ ) based on number of tuberculosis case in each health facility. Finally, DOTS User at the beginning of data collection was consecutively recruited provided that non double count in to the study until the intended 347 sample size fulfilled and interviewed in nearby facilities by using health professionals.

For qualitative Study purposive sampling was used conveniently to select participants for the in-depth interview to reflect TB treatment delay. To ensure reasonable spread, range of participants with respect to age, sex, and residence of 6 TB patients.

\subsection{Data Collection}

Prior to collect the required data, seven health facilities were selected from three rural district and one town administration. Questionnaire for interview was initially prepared in English and two individuals translated into Amharic and Hadiya local language, and then the final Version of the Amharic questionnaire was again translated into English by another person to check the consistency. A pre-tested structured questionnaire was administered to collect the intended data. Questions assessed, sociodemographics, major presenting symptoms of PTB, duration of major presenting symptoms and the date of first health care visit for each enrollee, Questions regarding knowledge of TB and perceived stigma was also included. With the permission of health workers at the DOTS clinic, patients coming to the centers for their treatment under DOTS was requested for consent and then interviewed for the required information. Information like date treatment started was recorded from the TB registers, lab register and TB treatment cards were cheeked to assure quality of data. Six Health professionals working in the study area interviewed the subjects after five minute following the TB treatment.

The questionnaire prepared to measure perceived stigma and knowledge were checked for their internal items consistency, the inter item reliability Cronbach alpha $=0.8$ and 0.73 respectively.

For qualitative: Interviews with TB patients were carried out independently by using in-depth interview guides which was prepared for patients. The principal investigator conducts the in-depth interview using a simple checklist of questions to be covered to collect the suggestions of the participants. The interview with different patients carried out until no newly emerging ideas emerged. The points were manually written by both the principal investigator and one other note taker. For In-depth Interviews the questions were similarly translated into the local language, Hadiyigna and Amharic like that of the standard and structured questionnaire.

\subsection{Data Processing and Analysis}

Data were entered into Epi-data version 3.1 and exported in to SPSS version 21 for analysis. Descriptive statistics like frequency distributions, median, proportion and inter quartile ranges (IQRs) were used. All variables with p-value less than 0.25 in bi-variate analysis were considered as candidates for multiple logistic regressions analysis. Multiple logistic regressions were tested for possible predictor's variable to identify factor associated with TB treatment delay and to control for potential confounders. Statistical significance was taken as $p<0.05$. Presence of statistical association between variables was observed by cross-tabulation using Pearson- $\chi 2$ $\&$ Fisher exact tests. Model for adjustment \& model adequacy was checked by Hosmer \& Lemeshow-GOF-test at $\mathrm{p}$-value $>0.05$. For Qualitative part all interviews were tape recorded, translated verbally into English and transcribed by principal investigator. The in-depth interviews were transcribed into English together with the moderators to allow for more thorough interpretations of the answers. All transcripts were cross-checked by the moderator. Interviews were regularly transcribed the same day or the following morning. The interview process was finished when the team considered that data saturation had been achieved (no new emerging ideas). Finally, information was linked to its congruence with data obtained from quantitative findings.

\section{Result}

\subsection{Socio-demographic Characteristics}

Three hundred and Forty PTB patients with a response rate of $97.7 \%$ were enrolled from seven diagnostics and treatment centers. Seven were excluded (Five were diagnosed in other health facilities outside study facility and two severely ill) during study period. The median age was $34(\mathrm{IQR}=23-42)$. Based on their residence $26 \%$ of the participants reside in urban, $17 \%$ semi urban and $57 \%$ ruler areas and of which $87 \%$ settled within $10 \mathrm{~km}$ from public health facilities with a median of $3 \mathrm{~km}$. With regard to education $58 \%$ were unable to read and write, $11.2 \%$ were primary school and $20.2 \%$ are high school and $10.6 \%$ college and above. The respondent's income distribution showed that $61.4 \%$ had an income of below 500ETB per month. The average monthly income was 452ETB $($ UUSD=22ETB) (Table 1). 
Table 1. Selected socio demographic characteristics of the respondents among pulmonary tuberculosis at Hadiya zone health facilities, SNNPR, $2017(n=340)$.

\begin{tabular}{lll}
\hline Variables & Frequency & Percent \\
\hline Sex of the respondents & 173 & 50.9 \\
Male & 167 & 49.1 \\
Female & & \\
Age of the respondents & 109 & 32.4 \\
18-24 years & 110 & 32 \\
25-34 year & 68 & 20 \\
35-44 year & 53 & 15.6 \\
$>=45$ years & & \\
Marital status & 108 & 66.2 \\
Single & 225 & 31.8 \\
Married & 7 & 2.1 \\
Divorced & & \\
Educational status & 192 & 56.4 \\
Unable to read and write & 47 & 13.8 \\
Primary school & 67 & 20.2 \\
High school & 35 & 10.6 \\
Collegeand above & & \\
Religion & 210 & 61.9 \\
Protestant & 105 & 30.9 \\
Orthodox & 25 & 7.4 \\
Muslim & & 71.8 \\
Ethnicity & 244 & 19.7 \\
Hadiya & 67 & 3.5 \\
Gurage & 12 & \\
Amhara & & \\
\hline
\end{tabular}

\begin{tabular}{lll}
\hline Variables & Frequency & Percent \\
\hline Kembata & 27 & 7.9 \\
Estimated Income in ETB & & \\
$0-500 \mathrm{ETB} /$ month & 178 & 52.4 \\
$501-1000 \mathrm{ETB} /$ month & 75 & 22.1 \\
$>=1000 \mathrm{ETB} /$ month & 87 & 25.5 \\
Occupation & & \\
Farmer & 69 & 20.5 \\
Merchant & 99 & 29.5 \\
Non employed & 56 & 20.5 \\
Employed & 69 & 16.5 \\
Housewife & 34 & 10.1 \\
Students & 13 & 3.8 \\
\hline
\end{tabular}

$\mathrm{ETB}=$ Ethiopian birr.

\subsection{Perceived Stigma}

One hundred and ninety (58) \%) had low stigma and $199(42 \%)$ reported high stigma towards TB and delayed in seeking, TB treatment for more than 30 days since the onset of TB symptoms. The majority $(66 \%)$ of respondents believed that TB disease affect relation with other, $56 \%$ of respondents felt that Ashamed of having TB disease, $66 \%$ of respondent were perceived that $\mathrm{TB}$ patients prefer to live isolated to other people( Table: 2 ).

Table 2. Perceived stigma of Respondents of Pulmonary tuberculosis at Hadiya zone public health facilities, SNNPR, 2017.

\begin{tabular}{lll}
\hline Perceived Stigma items $(\mathbf{n}=\mathbf{9})$ & Above mean(experienced stigma) & Below mean(didn't experienced stigma) \\
\hline I amashamed of having TB disease. & $190(56 \%)$ & $148(44 \%)$ \\
Having diagnosed to TB I have to hide other people & $142(42 \%)$ & $198(58 \%)$ \\
TB diseases affect relation with other people. & $225(66 \%)$ & $115(34 \%)$ \\
TB disease is very costly due to its long duration of treatment & $138(41 \%)$ & $202(59 \%)$ \\
I prefer to live isolated since diagnosed to TB disease & $203(60 \%)$ & $137(40 \%)$ \\
TB diseases can affect work performance & $135(40 \%)$ & $205(60 \%)$ \\
TB disease can affect family relation. & $138(40.6 \%$ & $202(59.4)$ \\
TB disease is poverty disease & $135(40 \%)$ & $205(60 \%)$ \\
Having diagnosed to TB, I feel inferiority complex to my family & $135(39.6 \%)$ & $205(59.4 \%)$ \\
\hline
\end{tabular}

Finding from Qualitative study showed, a33 year, female key informants, states "When men get sick the source of support from all family members like the in-laws and others. Male gets support all the time from the family. In fact if the woman gets sick, even if in that situation the females are expected to perform daily task. The other women age 42 supports this idea, Women is always busy with something so they don't think about themselves, they think about others. Men are quicker than women to seek care and find out about the disease. Women are busy with the family and may think it is of no importance, they always come late.

\subsection{Knowledge about Tuberculosis}

Most of the respondents were not aware of the causes of TB. Only $41.7 \%$ of the patients however had knowledge about the TB infection causing micro-organism most of the patients $(56 \%)$, understood that $\mathrm{TB}$ is transmitted mainly through air droplets resulting from coughing by a TB patient while $22 \%$ believed that TB infection is through punishment and $18 \%$ is unavoidable. Majority $(68 \%)$ of the patients had said that TB can be diagnosed by sputum examination followed by chest x-ray (56\%) and some patients recognizing signs and symptoms, while the remainder had no knowledge about the causes. Most of the patients did not know about the correct prevention of $\mathrm{TB}$ as shown below. Regarding the overall knowledge of participants on the causes, transmission, prevention, treatment and outcome of TB, $60.4 \%$ of participants had poor knowledge and remaining had good knowledge (Table 3). 
Table 3. Knowledge on TB among PTB patients at Hadiya zone health facilities, SNNPR, 2017.

\begin{tabular}{lll}
\hline \multirow{2}{*}{ Items } & Knowledge (\%) & No \\
\cline { 2 - 3 } & Yes & 42.4 \\
\hline Do you know what TB(samba nekarissa) is? & 57.6 & 52 \\
TB (samba nekarissa) is a serious disease & 48 & 45.6 \\
What in your opinion cause TB? & 54.4 & 39 \\
What are the symptoms of someone infected with TB? & 61 & 44.4 \\
How can a person get infected (Transmission) & 55.6 & $49 \%$ \\
Do you know how TB is diagnosed? & 51 & 45 \\
TB will require a longer treatment to be cured after treatment & 55 & 55 \\
TB treatment and diagnosis isfree ofcharge & 45 & 62 \\
Tuberculosis diseaseis killer disease & 38 & 44.4 \\
TB affect HIV/AIDS prognosis & 55.6 & 45.9 \\
Do you have opinion TB prevention methods & 54.1 & 49 \\
What are TB prevention Methods & 51 & 60.3 \\
TB disease is poverty disease & 39.7 & \\
\hline
\end{tabular}

\subsection{Patient Factors and Tuberculosis Treatment Delay}

Nearly half $(49.1 \%)$ had sought care in the public health facilities after 30 days of onset of symptoms. The median patient delay was 31 (IQR 28-32). The longest patient delay was 120 recorded were among patients who had practicing traditional healer. Binary logistics regression analysis through backward likelihood ratio methods employed to assess the relative effect of the explanatory variables on the dependent variable after checking bivariate analysis. On the
Bivariate analysis of socio-demographic factors unable to read and write and primary school had a significant increase the risk of delay in seeking health care of TB treatments. Respondents with poor knowledge about TB, and those with prior attendance at traditional healers, self-medication, nearby dispensary local pharmacy and financial constraints were associated with patient delay in seeking health care of TB treatment (Table 4).

Table 4. Summary of Bi-variate and Multivariate analysis of factors associated with delay in seeking health care among Pulmonary TB patients at Hadiya Zone, SNNPR, 2017.

\begin{tabular}{|c|c|c|c|c|c|}
\hline Variables & Patient delay $>\mathbf{3 0}$ days & Patient delay $<30$ days & $\operatorname{COR}(95 \% \mathrm{CI})$ & $\operatorname{AOR}(95 \% \mathrm{CI})$ & p-value \\
\hline \multicolumn{6}{|l|}{ Education Status } \\
\hline Unable to read and write & 168 & 68 & $5.7(2.5712 .95)$ & $6(3.1121 .36)$ & 0.001 \\
\hline Primary & 22 & 10 & $1.07(0.353 .05)$ & $1.76(0.535 .84)$ & 0.350 \\
\hline High school & 56 & 32 & $1.2(0.4873 .08)$ & $1.861(0.5864 .82)$ & 0.334 \\
\hline College and above & 23 & 10 & 1 & 1 & \\
\hline \multicolumn{6}{|l|}{ Knowledge } \\
\hline Poor & 131 & 75 & $4.4(2.6766 .815)$ & $4(2.2836 .863)$ & 0.001 \\
\hline Good & 38 & 96 & 1 & 1 & \\
\hline \multicolumn{6}{|l|}{ Informal treatment sources } \\
\hline Traditional healers (Yes) & 81 & 68 & $1.39(0.902 .14$ & $1.23(1.113 .94)$ & 0.022 \\
\hline $\mathrm{NO}$ & 88 & 103 & 1 & 1 & \\
\hline \multicolumn{4}{|l|}{ Pharmacy shop dispensary } & $2(1.014 .05)$ & 0.049 \\
\hline NO & 113 & 132 & 1 & 1 & $1: 00$ \\
\hline NO & 66 & 127 & 1 & 1 & \\
\hline Resorts visit(Yes) & 22 & 32 & $0.67(0.4311 .45)$ & $0.84(0.371 .89)$ & 0.681 \\
\hline No & 147 & 143 & $1: 00$ & $1 ; 00$ & \\
\hline Financial constraints(Yes & 54 & 28 & 2.3(1.413..99 & $2(1.1103 .96)$ & 0.022 \\
\hline No & 116 & 142 & 1 & 1 & \\
\hline \multicolumn{6}{|l|}{ Residence } \\
\hline Rural & 106 & 90 & $0.5\left(\begin{array}{lll}0.30 & 0.85\end{array}\right)$ & $0.44(0.230 .83)$ & 0.784 \\
\hline Urban & 34 & 56 & 0.9 & $1.078(0.5142 .263$ & 0.842 \\
\hline Semi urban & 29 & 25 & 1 & 1 & \\
\hline \multicolumn{6}{|l|}{ Walking distance } \\
\hline$>10 \mathrm{KM}$ & 14 & 30 & $2.4(1.234 .75)$ & $1.39(0.762 .52)$ & 0.279 \\
\hline$<10 \mathrm{~km}$ & 157 & 139 & 1 & 1 & \\
\hline
\end{tabular}

On multivariable Logistics analysis, being unable to read and write were associated with patient delay in seeking health care of TB treatments. The odds of delay in seeking health care of TB treatments for those who unable to read 
and write were 6 times higher than the odds of respondents those attend college and above Education with [AOR: 6.05, $95 \%$ CI 2.2836.863]. The odds of delay in seeking health care of TB treatments for who had financial constraints (transportation cost) were 2 times higher than the odds of their counterparts with [AOR: 2, 95\%CI (1.1103.945). The odds of delay in seeking health care of tuberculosis treatments for respondents with poor knowledge about tuberculosis treatment were 4 fold times higher than the odds of delay in seeking health care of respondents with good knowledge about tuberculosis with [AOR: 3.958, 95\%CI 2.2836.861]. The odds of delay for TB treatment for respondents with self-medication about TB treatment were 2 times higher than the Odds of delay in tuberculosis treatment who didn't practice self-medication (AOR:2.121, 95\%CI (1.1.1443.933).

Prior Attendance at nearby drug store, and traditional healers [AOR: 1.23(1.113.94) had also shown Association of patient delay in seeking health care of TB treatments.

From Qualitative studies of IDI, two health professional, age of 30 and different sex states "The TB patients usually come from very poor families, and they usually live under poor conditions. Popular rules state that the man should get treatment before the woman since men is the pillar of the household. Other person supports these ideas, in the rural areas; the status of women is lower than that of men. When men get TB, all family resources may be spent, but that didn't do for women. So first of all the females hides her illness and then maybe the family does not support her financially so woman has more difficulties to visit health facilities.

\section{Discussion}

Early diagnosis of disease and prompt initiation of treatment is essential for an effective TB control program. Delay in diagnosis and treatment may worsen the disease, increase risk of poor clinical outcome, including death and enhance transmission of TB in the community. The finding of this study indicated that there is a substantial prolonged delay in health care seeking of pulmonary TB after the onset of symptoms. World Health organization (WHO) classifies unexplained cough with or without other symptoms lasting 2-3 weeks as TB suspect and the patients will seek treatments within the given period [13]. However, studies added extra margin of 7 days to take 30 days as cutoff point to assess magnitude of patient delay and factors: a delay $>30$ days [14-16].

The median patients delay was 31 days. $49.1 \%$ of the patients delayed beyond the median. 128 (37.6\%) of smear positive patients had a delay more than 30 days. The prevalence of patient delay in this study was $49.1 \%$ which is consistent with studies done in Oromia region and Tigray $(49.7 \%, 53 \%$ respectively $[14,17]$. But the result of this study was lower than studies done in Somali region, Ethiopia (87\%) [18]. The possible reason for this gap might be due to the fact that in Somalia region, where majority nomadic people were lives in dispersed location and inaccessible to modern medical health care compare to Hadiya zone, SNNPR.

The median patient delay in this study was 31 days. This is in consistent with studies from Bahir Dar, Arsi zone and Tigray Region of Ethiopia [18, 14, 19], These findings are consistent with previous studies from East Mediterranean of WHO studies in Iraq (median, 31 days), Syria (median 31 days), and Somalia. However, the result of the same study in east Mediterranean in Pakistan (median, 9 days), Egypt (median, 12 days) showed that TB patients were more less delayed than in this study [20]. The possible explanation for differences in delay could probably be explained by the socio-cultural factor, low socio economic status, low level of knowledge and awareness of the disease and lack of information about availability of free TB treatment.

Unable to read and write in educational status was found to be predictor of patient delay, this finding which is consistent with result from Ethiopia [12, 21, 22]. This could be due to the fact that those patients with no education have poorer knowledge About TB and thus 4 times less likely to seek health care in health facilities at the earliest time [AOR:3.95, 95\% CI (2.2836.863)].

Poor knowledge of TB is associated with patient delay. The association between poor knowledge of TB and patient delay is very strong as is also the case in other studies from Bahir Dar, Tigray, Somali and Uganda [20, 22], [23, 24]. Financial constraints for transportation were associated with patient delay in this study. This is in line with report from Kampala [12, 25]. Self-treatment (Medication) was found to be determinants of patient delay. This is similar with findings from Afar Ethiopia, Georgia [12, 25]. This was supported by Qualitative study finding.

A 38 years old female who was living in rural area said that "most orthodox Christian followers went to churches where different faith based service is provided as initial treatment and stays for a period of time like one week determined by church leaders".

This finding indicated $56.4 \%$ of TB patients had sought care in health facilities after visited alternative source of treatment (pray church, traditional healer and private drug store) and this study was consistent with study done in Tigray [19], Amhara [18]. The possible explanation using of informal therapies might be related to religious beliefs of patients, in which about $87 \%$ of the study participants were Protestant and orthodox Christians. Church, the most predominant religious FaithBased institution in the study area.

In this study nearly half, $42 \%$ of the study participants had perceived stigma of TB on relationship with other, isolated to live other since diagnosed, social and family relationship. This study consistent with Gibe, south west Ethiopia [15], Perception of stigma might have great impact on the social, psychological and mental wellbeing of the victim and also their family. This was supported by the qualitative study, a 30 years old woman in the urban area said that "If TB status is disclosed to the relatives, it will results to isolation in the community. "The other person a 50 years old man in rural kebele expressed the idea by saying that "some people in the 
community reduce social interaction with $\mathrm{TB}$ patients because it is difficult to identify whether they are free from HIV or not. This indicates negative attitude towards TB treatment.

\section{Conclusion}

This study found significant association between TB treatment delay and associated factors among PTB patients. There is substantial prevalence of patient delay in study area. Unable to read and write, Poor knowledge of TB, selftreatment, financial constraints and (transportation) were found to have association with patient delay. This may lead to continues existence of TB cases which probably leads to the emergence of MDRTB (Multi Drug Resistant TB). Therefore, it is suggested that, Poor knowledge of TB and self-treatment can be prevented by community based interventions. Health professionals should be supported to strengthen the activities of health education and promotion. Through a well-designed IEC/BCC strategy for TB might improve the TB control program.

\section{Ethical Approval}

Ethical approval or clearance letter obtained from institutional reviewing board (IRB), Jimma University, institute of health. Permission letter obtained from Hadiya zone health department and respective district hospitals and Hosanna town health office. Verbal consent was obtained from each study subjects and participants told that they had full right to participate or refuse participation in the study.

\section{Availability of Data and Materials}

(1) The SPSS data used to support the findings of this study are included within the article.

(2) The SPSS data used to support the findings of this study are available from the corresponding author upon request.

\section{Competing Interests}

All authors declare that they have no competing interests.

\section{Acknowledgements}

We would like to forward our thanks to Jimma University for funding this study; for all study participants, data collectors and supervisors.

\section{Authors' Contributions}

GG had made substantial contributions to conception and design, acquisition of data, analysis and interpretation of data, and $\mathrm{BE}, \mathrm{LA}$, and $\mathrm{MC}$ had revised the paper critically for important intellectual content starting from proposal development to manuscript preparation. All authors read and approved the final manuscript.

\section{References}

[1] WHO, Global Tuberculosis Report 2013. World Health Organization.

[2] FMOH, (2012) Guidelines for clinical and programmatic management of TB, leprosy and TB/HIV in Ethiopia. FMOH, Addis Ababa.

[3] Tsegaye D, A. E., Mesele T, Tadesse T, etal, Delay in Seeking Health Care and associated Factors among Pulmonary Tuberculosis patients in North Wollo Zone, Northeast Ethiopia: /institutionBased crossectional Study. iMedPub Journals 2016 7(3) 1-7.

[4] WHO, Global Tuberculosis control.2010, WHO report.

[5] WHO, Global Tuberculosis Report 2014a, Geneva: World Health Organization(2014a). WHO report.

[6] WHO, World Health Organization (WHO) Global Tuberculosis Report, 2015, WHO report.

[7] Abebe G, Deribew A, and Apers L, Knowledge, health seeking behavior and perceived stigma towards tuberculosis among tuberculosis suspects in a rural community in southwest Ethiopia. PLOS ONE 5(10).

[8] FDREMOH, FDRE, Guideline for the national TB Leprosy Control Programme in Ethiopia Addis Ababa. Federal Ministry of Health Ethiopia, 2007.

[9] Dye C, W. B., (2012) The population dynamics and control of tuberculosis. PubMedGoogle Scholar 328(5980).

[10] Engeda EH, D. B., WoretaHK, KelkayMM, Ashenafie TD, eatal, (2016) Health Seeking Behaviour and Associated Factors among Pulmonary Tuberculosis Suspects in Lay Armachiho District, Northwest Ethiopia:Tuberc Res Treat.

[11] Yimer S, B. G., Alen G, Diagnostic and treatment delay among pulmonary tuberculosis patients in Ethiopia: a cross sectional study. BMC Infectious Diseases2005, 5(112).

[12] Demissie M, L. B., Berhane Y, (2002) Patient and health service delay in the diagnosis of pulmonary tuberculosis in Ethiopia. BMC Public Health 22(23) 1-23.

[13] Belay M, B. G., Ameni G, Abebe F, etal, (2012)Diagnostic and treatment delay among Tuberculosis patients in Afar Region, Ethiopia: A cross-sectional study. BMC Public Health, 12(369) PP1-23.

[14] Gebeyehu E, A. M., Abeje G,, (2014) Factors Associated with Patient's Delay in Tuberculosis Treatment in Bahir Dar City Administration, Northwest Ethiopia. BioMed Research International, Article ID: 701429.

[15] Mesfin MM, T. T., Tareke IG, Kifle YT, Karen WH, Richard MJ, etal, (2005)Delays and care seeking behavior among tuberculosis patients in Tigray of northern Ethiopia., Ethiopian Journal of Health Development 19(12) 7-12.

[16] Takarinda, H., AD, Nyathi B, Ngwenya M, Mutasa-ApolloT, SandyC, etal., Tuberculosis treatment delays and associated factors within the Zimbabwe national tuberculosis programme, 2016. MC Public Health 15(29) pp1-12. 
[17] Cambanis A, Y. M., Ramsay A, Squire SB, ArbideI, Cuevas L, etal, (2016) Rural poverty and delayed presentation to tuberculosis services in Ethiopia, Tropical Medicine and International Health21(1365-3156).

[18] WHO, An in-depth analysis of the health-seeking behaviour of patients and health system responce in seven countries of eastern Meditrenian Region.2009WHO swizerland, Geneva. p. $1-48$.

[19] Liam CK, T. B., Delay in the diagnosis and treatment of pulmonary tuberculosis in patient attending a University Teaching Hospital.1997, NT J TUBERC LUNG DIS 1(326332).

[20] Salaniponi FM, H. A., Banda HJ,, Care seeking behaviour and diagnostic processes in patients with smear positive pulmonary tuberculosis in Malawi. INT J TUBERC LUNG DIS 1991 4(327-332).

[21] Kansiime C, K. S., Levi M, Asiimwe BB, KatambaA, etal,
Health Service Delay among Pulmonary Tuberculosis Patients Presenting to a National Referral Hospital, Kampala, Uganda: A Cross Sectional Study. The Pan African Medical Journal, 2013, 15(84).

[22] Yimer S, G. Bjune, and Alene G, Diagnostic and treatment delay among pulmonary tuberculosis patients in Ethiopia: a cross sectional study.2005, BMC Infectious Diseases2005, 5(112) PP 1-8.

[23] Hamza A, D. M., Gare S, Teshome G,, (2015) Delay in Tuberculosis Diagnosis amongTuberculosis Patients at the Three Hospitals: Asella, Robe and Abomsa of Arsi Zone, Oromia Regional State, Open Access Library journal(2).

[24] Gele AA, B. G., AbebeF,, (2009) Pastoralism and delay in diagnosis of TB in Ethiopia. BMC Public Health 9(5).

[25] Yimer S, Bjune G, and Alene G, Diagnostic and treatment delay among pulmonary tuberculosis patients in Ethiopia: a cross sectional study.2005, BMC Infect Dis, 5(112). 